\title{
Kohn-Sham composition of the excitations:
} \section{BFPF}

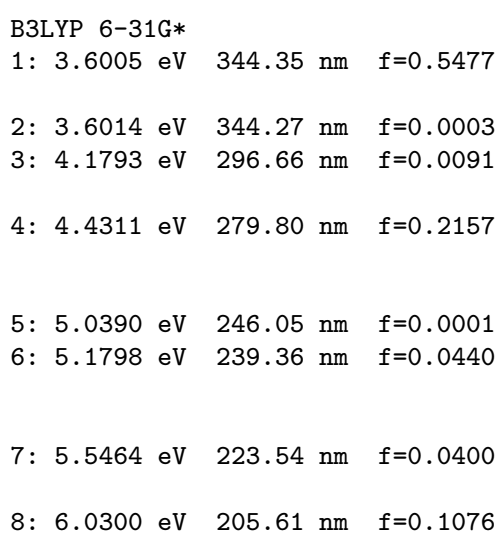




\section{$\mathrm{BFP}_{\delta}$}

B3LYP $631 \mathrm{G*}$

1: $3.5926 \mathrm{eV} \quad 345.11 \mathrm{~nm} \quad f=0.6090$

$\begin{array}{llr}50 \rightarrow 5 & \\ 49 \rightarrow 51 & 0.58772 \\ 48 \rightarrow 51 & 0.69337 \\ 47 \rightarrow 51 & 0.65289 \\ 46 \rightarrow 51 & 0.67451 \\ 44 \rightarrow 51 & 0.69037 \\ 45 \rightarrow 51 & -0.25030 \\ 50 \rightarrow 52 & 0.63166 \\ 43 \rightarrow 51 & -0.13849 \\ 45 \rightarrow 51 & 0.36193 \\ 50 \rightarrow 52 & 0.13608 \\ 50 \rightarrow 53 & 0.54264\end{array}$

B3LYP $631+\mathrm{G} *$

1: $3.5076 \mathrm{eV} \quad 353.47 \mathrm{~nm} \quad f=0.6197$

$50 \rightarrow 51$

0.59302

2: $3.8023 \mathrm{eV} \quad 326.08 \mathrm{~nm} \quad f=0.0003$

$49 \rightarrow 51$

0.69406

3: $4.3172 \mathrm{eV} \quad 287.18 \mathrm{~nm} \quad f=0.0938$

$46 \rightarrow 51$

0.10003

$48 \rightarrow 51-0.65588$

4: $4.5119 \mathrm{eV} \quad 274.79 \mathrm{~nm} \quad f=0.0000$

$47 \rightarrow 51$

0.70169

5: $4.9033 \mathrm{eV} \quad 252.86 \mathrm{~nm} \quad f=0.0000$

$50 \rightarrow 52$

0.69068

$50 \rightarrow 53 \quad-0.10947$

$46 \rightarrow 51 \quad 0.67294$

6: $4.9567 \mathrm{eV} \quad 250.13 \mathrm{~nm} \quad f=0.0037$

$50 \rightarrow 52$

0.67294
0.11173

$50 \rightarrow 53 \quad 0.68561$

8: $5.3995 \mathrm{eV} \quad 229.62 \mathrm{~nm} \quad f=0.0003$

$44 \rightarrow 51$

0.68970

B3LYP 631+G* PCM (Methanol)

1: $3.3790 \mathrm{eV} \quad 366.93 \mathrm{~nm} \quad f=0.6904$

$50 \rightarrow 51 \quad 0.60831$

$48 \rightarrow 51 \quad 0.69571$

$49 \rightarrow 51 \quad 0.66324$

: 3.9321 eV $315.31 \mathrm{~nm} \quad f=0.0004$

0.66324
0.70017

4: $4.8473 \mathrm{eV} \quad 255.78 \mathrm{~nm} \quad f=0.0000$

$46 \rightarrow 51$

0.67136

5: $5.0196 \mathrm{eV} \quad 247.00 \mathrm{~nm} \quad f=0.0071$

$47 \rightarrow 51$

$50 \rightarrow 52 \quad 0.68597$

$50 \rightarrow 53 \quad 0.13232$

7: $5.4121 \mathrm{eV} \quad 229.09 \mathrm{~nm} \quad f=0.0002$

$44 \rightarrow 51$

0.69130

8: $5.6571 \mathrm{eV} \quad 219.17 \mathrm{~nm} \quad f=0.0119$

0.37258

$50 \rightarrow 54 \quad 0.57201$

$50 \rightarrow 55 \quad-0.11529$ 


\section{$\mathrm{BFP}_{\epsilon}$}

B3LYP $631 \mathrm{G*}$

1: $3.6186 \mathrm{eV} \quad 342.63 \mathrm{~nm} f=0.0003$

2: $3.7410 \mathrm{eV} 331.42 \mathrm{~nm} \quad f=0.4660$

3: $4.5545 \mathrm{eV} \quad 272.22 \mathrm{~nm} \quad \mathrm{f}=0.2528$

4: $4.7682 \mathrm{eV} \quad 260.02 \mathrm{~nm} \quad f=0.0004$

5: $5.1759 \mathrm{eV} \quad 239.54 \mathrm{~nm} \quad f=0.0011$

6: $5.2835 \mathrm{eV} \quad 234.66 \mathrm{~nm} \quad \mathrm{f}=0.1152$

7: $5.5849 \mathrm{eV} \quad 222.00 \mathrm{~nm} \quad \mathrm{f}=0.0216$

8: $6.0279 \mathrm{eV} \quad 205.68 \mathrm{~nm} \quad \mathrm{f}=0.0022$

$\begin{array}{rlr}49 \rightarrow 51 & 0.69435 \\ 46 \rightarrow 51 & -0.10210 \\ 48 \rightarrow 51 & -0.17489 \\ 50 \rightarrow 551 & 0.58457 \\ 48 \rightarrow 51 & 0.64376 \\ 50 \rightarrow 51 & 0.13953 \\ 50 \rightarrow 55 & 0.12008 \\ 45 \rightarrow 51 & -0.14722 \\ 47 \rightarrow 51 & 0.68074 \\ 45 \rightarrow 551 & 0.67501 \\ 47 \rightarrow 51 & 0.15666 \\ 46 \rightarrow 51 & 0.60027 \\ 50 \rightarrow 55 & -0.32320 \\ 46 \rightarrow 51 & 0.30158 \\ 50 \rightarrow 52 & 0.58223 \\ 43 \rightarrow 51 & 0.14526 \\ 44 \rightarrow 51 & -0.28350 \\ 50 \rightarrow 53 & 0.61223\end{array}$

B3LYP $631+\mathrm{G} *$

1: $3.6396 \mathrm{eV} \quad 340.65 \mathrm{~nm} \quad f=0.0003$

$49 \rightarrow 51$

$48 \rightarrow 51$

$50 \rightarrow 51$

$48 \rightarrow 51$

3: $4.4888 \mathrm{eV} \quad 276.21 \mathrm{~nm} \quad f=0.2398$

$50 \rightarrow 51$

$50 \rightarrow 54$

4: $4.6516 \mathrm{eV} \quad 266.54 \mathrm{~nm} \quad f=0.0001$

$50 \rightarrow 52$

$45 \rightarrow 51$

$47 \rightarrow 51$

$50 \rightarrow 53$

$46 \rightarrow 51$

$50 \rightarrow 54$

$45 \rightarrow 51$

$47 \rightarrow 51$

0.69492

$-0.16163$

0.59195

0.64730

0.12779

$-0.12080$

0.69865

$-0.14510$

0.68111

0.69374

0.38438

0.56629

0.67587

8: $5.1900 \mathrm{eV} \quad 238.89 \mathrm{~nm} \quad f=0.0008$

0.15410

B3LYP 631+G* PCM (Methanol)

1: $3.5596 \mathrm{eV} \quad 348.31 \mathrm{~nm} \quad f=0.6608$

$50 \rightarrow 51$

0.61369

2: $3.9202 \mathrm{eV} \quad 316.27 \mathrm{~nm} \quad f=0.0003$

$49 \rightarrow 51$

0.69524

$48 \rightarrow 51 \quad 0.66280$

4: $4.9130 \mathrm{eV} \quad 252.36 \mathrm{~nm} \quad \mathrm{f}=0.0007$

$46 \rightarrow 51$

0.69207

5: $5.1153 \mathrm{eV} \quad 242.38 \mathrm{~nm} \quad \mathrm{f}=0.0712$

$47 \rightarrow 51$

0.65761

$50 \rightarrow 53 \quad 0.17638$

6: $5.2169 \mathrm{eV} \quad 237.66 \mathrm{~nm} \quad f=0.0000$

$50 \rightarrow 52$

0.68527

$50 \rightarrow 54-0.11978$

$45 \rightarrow 51 \quad 0.68544$

7: $5.4736 \mathrm{eV} \quad 226.51 \mathrm{~nm} \quad f=0.0010$

$47 \rightarrow 51$

$50 \rightarrow 53$

$-0.15147$ 


\section{CFP}

B3LYP $631 \mathrm{G*}$

1: $3.4172 \mathrm{eV} \quad 362.82 \mathrm{~nm} \quad f=0.6248$

2: $3.7472 \mathrm{eV} \quad 330.87 \mathrm{~nm} \quad f=0.0003$

3: $3.9348 \mathrm{eV} \quad 315.10 \mathrm{~nm} \quad f=0.0099$

4: $4.1268 \mathrm{eV} \quad 300.44 \mathrm{~nm} \quad f=0.0045$

$5: 4.4036 \mathrm{eV} \quad 281.55 \mathrm{~nm} \quad f=0.0715$

6: $4.9438 \mathrm{eV} \quad 250.79 \mathrm{~nm} \quad \mathrm{f}=0.0972$

7: $5.0441 \mathrm{eV} \quad 245.80 \mathrm{~nm} \quad \mathrm{f}=0.1642$

8: $5.2198 \mathrm{eV} \quad 237.53 \mathrm{~nm} \quad f=0.0002$

\begin{tabular}{|c|c|c|}
\hline 63 & -> 64 & 0.60567 \\
\hline 60 & -> 64 & 0.69289 \\
\hline 61 & -> 64 & -0.13332 \\
\hline 62 & -> 64 & 0.63597 \\
\hline 63 & $\rightarrow 65$ & 0.23492 \\
\hline 59 & -> 64 & 0.11013 \\
\hline 61 & -> 64 & 0.65056 \\
\hline 62 & -> 64 & 0.15565 \\
\hline 62 & $\Rightarrow 64$ & -0.19556 \\
\hline 63 & $\rightarrow 65$ & 0.63130 \\
\hline 59 & -> 64 & 0.40896 \\
\hline 51 & -> 65 & -0.11680 \\
\hline 62 & $\rightarrow>65$ & -0.39893 \\
\hline 63 & $\rightarrow$ & 0.36479 \\
\hline 59 & -> 64 & 0.51797 \\
\hline ? & $\rightarrow 65$ & $0.1817 \varepsilon$ \\
\hline & $\Rightarrow 66$ & $-0.3911 s$ \\
\hline & -> 64 & 0.6880 \\
\hline
\end{tabular}

B3LYP $631+G *$

1: $3.3165 \mathrm{eV} \quad 373.84 \mathrm{~nm} \quad f=0.5986$

2: $3.7715 \mathrm{eV} \quad 328.74 \mathrm{~nm} \quad f=0.0004$

3: $3.8674 \mathrm{eV} \quad 320.59 \mathrm{~nm} \quad f=0.0142$

4: $4.0575 \mathrm{eV} \quad 305.57 \mathrm{~nm} \quad \mathrm{f}=0.0048$

5: $4.2520 \mathrm{eV} \quad 291.59 \mathrm{~nm} \quad f=0.0668$

6: $4.4951 \mathrm{eV} \quad 275.82 \mathrm{~nm} \quad f=0.0002$

7: $4.7469 \mathrm{eV} \quad 261.19 \mathrm{~nm} \quad f=0.0000$

8: $4.8312 \mathrm{eV} \quad 256.63 \mathrm{~nm} \quad f=0.0346$

$$
\begin{aligned}
& 63 \rightarrow 64 \quad 0.61074 \\
& 60 \rightarrow 64 \quad 0.69381 \\
& 61 \rightarrow 64 \quad-0.13907 \\
& 62 \rightarrow 64 \quad 0.63142 \\
& 63 \rightarrow 65 \quad 0.24261 \\
& 59 \rightarrow 64 \quad 0.11059 \\
& 61 \rightarrow 64 \quad 0.65029 \\
& 62 \rightarrow 64 \quad 0.16174 \\
& 62 \rightarrow 64 \quad-0.20798 \\
& 63 \rightarrow 65 \quad 0.63230 \\
& 63 \rightarrow 66 \quad 0.69873 \\
& 63 \rightarrow 67 \quad 0.69247 \\
& 59 \rightarrow 64 \quad 0.28853 \\
& 61 \rightarrow 65 \quad-0.10967 \\
& 61 \rightarrow 68 \quad-0.10596 \\
& 62 \rightarrow 65 \quad-0.43267 \\
& 63 \rightarrow 68 \quad 0.44212
\end{aligned}
$$

B3LYP 631+G* PCM (Methanol)

1: $3.1547 \mathrm{eV} \quad 393.02 \mathrm{~nm} \quad f=0.6987$

2: $3.7427 \mathrm{eV} \quad 331.27 \mathrm{~nm} \quad f=0.0267$

3: $3.9701 \mathrm{eV} \quad 312.30 \mathrm{~nm} \quad f=0.0007$

4: $3.9740 \mathrm{eV} \quad 311.99 \mathrm{~nm} \quad f=0.0095$

5: $4.3304 \mathrm{eV} \quad 286.31 \mathrm{~nm} \quad \mathrm{f}=0.0737$

$6: 4.7813 \mathrm{eV} \quad 259.31 \mathrm{~nm} \quad f=0.2220$

$63 \rightarrow 64 \quad 0.62483$

$61 \rightarrow 64 \quad 0.10043$

$62 \rightarrow 64 \quad 0.67119$

$63 \rightarrow 65 \quad 0.13674$

$60 \rightarrow 64 \quad 0.68669$

$61 \rightarrow 64 \quad-0.10715$

$59 \rightarrow 64 \quad-0.12439$

$60 \rightarrow 64 \quad 0.11435$

$61 \rightarrow 64 \quad 0.64315$

$62 \rightarrow 64 \quad-0.11369$

$62 \rightarrow 64 \quad-0.10780$

$63 \rightarrow 65 \quad 0.66149$

$59 \rightarrow 64 \quad 0.60634$

$62 \rightarrow 65 \quad-0.28988$

$63 \rightarrow 67 \quad 0.13267$

$63 \rightarrow 66 \quad 0.68023$

$63 \rightarrow 68 \quad-0.13088$

$63->71 \quad-0.10656$ 


\section{GFP $_{\mathrm{N}}$}

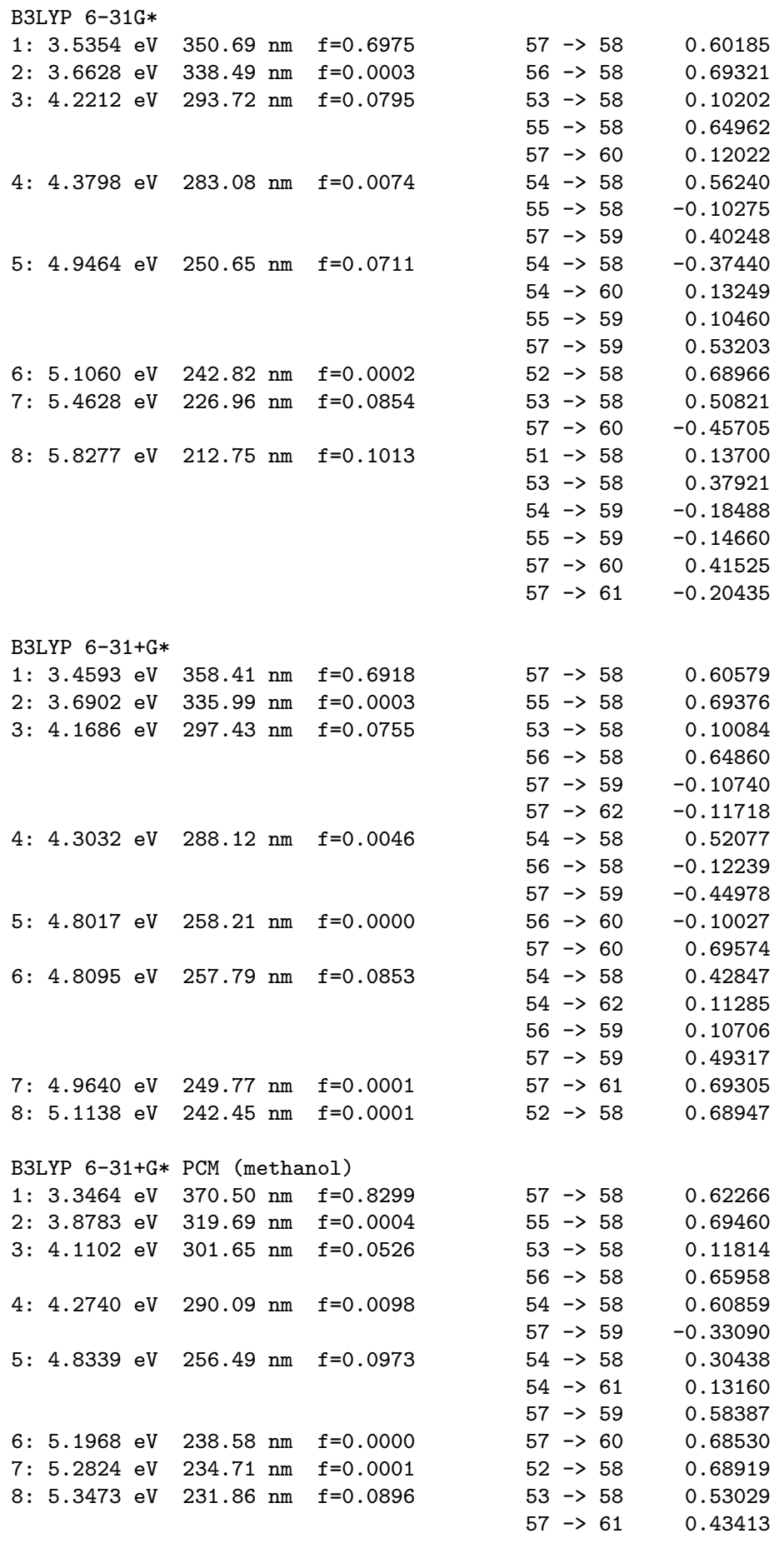




\section{GFP $_{\mathrm{A}}$}

B3LYP 6-31G*

1: $3.0914 \mathrm{eV} \quad 401.06 \mathrm{~nm} \quad f=0.0000$

2: $3.1942 \mathrm{eV} \quad 388.15 \mathrm{~nm} \quad f=0.9840$

3: $4.1633 \mathrm{eV} \quad 297.80 \mathrm{~nm} \quad f=0.0004$

4: $4.3215 \mathrm{eV} \quad 286.90 \mathrm{~nm} \quad f=0.0031$

5: $4.3781 \mathrm{eV} \quad 283.19 \mathrm{~nm} \quad f=0.0110$

6: $4.4777 \mathrm{eV} \quad 276.89 \mathrm{~nm} \quad \mathrm{f}=0.0564$

7: $4.7508 \mathrm{eV} \quad 260.97 \mathrm{~nm} \quad f=0.0718$

8: $4.9796 \mathrm{eV} \quad 248.98 \mathrm{~nm} \quad f=0.0001$

\begin{tabular}{|c|c|c|}
\hline 56 & -> 58 & 0.68715 \\
\hline 57 & -> 58 & 0.57114 \\
\hline 53 & -> 58 & 0.69617 \\
\hline 52 & -> 58 & 0.13186 \\
\hline 54 & -> 58 & 0.12181 \\
\hline 55 & -> 58 & 0.62124 \\
\hline 57 & -> 62 & -0.16694 \\
\hline 54 & -> 58 & 0.48722 \\
\hline 57 & -> 59 & -0.46669 \\
\hline 54 & -> 58 & 0.46736 \\
\hline 57 & $\rightarrow 59$ & 0.46943 \\
\hline 52 & $\rightarrow 58$ & 0.14138 \\
\hline 57 & $\rightarrow 60$ & 0.65497 \\
\hline 56 & $\rightarrow 59$ & 0.69072 \\
\hline 56 & 60 & 0.12056 \\
\hline
\end{tabular}

B3LYP 6-31+G*

1: $2.9725 \mathrm{eV} \quad 417.10 \mathrm{~nm} \quad f=0.0001$

2: $3.0762 \mathrm{eV} \quad 403.04 \mathrm{~nm} \quad f=0.9867$

$\begin{array}{lll}57 & \rightarrow 59 & 0.70341\end{array}$

3: $3.2146 \mathrm{eV} \quad 385.69 \mathrm{~nm} \quad f=0.0000$

4: $3.5813 \mathrm{eV} \quad 346.20 \mathrm{~nm} \quad f=0.0007$

$57 \rightarrow 58$

0.57913

$56 \rightarrow 58 \quad 0.68818$

5: $3.8618 \mathrm{eV} \quad 321.05 \mathrm{~nm} \quad f=0.0001$

$57 \rightarrow 60 \quad 0.70378$

$57 \rightarrow 61 \quad 0.69751$

6: $4.0540 \mathrm{eV} \quad 305.83 \mathrm{~nm} \quad f=0.0003$

$\begin{array}{lll}57 \rightarrow 62 & 0.70178\end{array}$

7: $4.1520 \mathrm{eV} \quad 298.61 \mathrm{~nm} \quad f=0.0010$

$57 \rightarrow 64 \quad 0.69466$

$54 \rightarrow 58 \quad-0.21023$

8: $4.1704 \mathrm{eV} \quad 297.29 \mathrm{~nm} \quad f=0.0525$

$57 \rightarrow 63 \quad 0.63386$

B3LYP 6-31+G* PCM (methanol) [optimized with 6-31G* PCM (methanol)]

1: $3.1490 \mathrm{eV} \quad 393.72 \mathrm{~nm} \quad \mathrm{f}=0.9349 \quad 57 \rightarrow 58 \quad 0.58802$

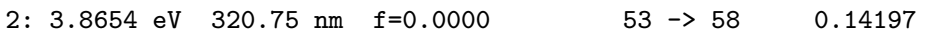

$55 \rightarrow 58 \quad 0.67893$

3: $4.0528 \mathrm{eV} \quad 305.92 \mathrm{~nm} \quad f=0.0035$

4: $4.0715 \mathrm{eV} \quad 304.52 \mathrm{~nm} \quad f=0.0082$

$53 \rightarrow 58 \quad 0.58184$

$55 \rightarrow 58 \quad-0.12767$

$56 \rightarrow 58-0.33137$

$52 \rightarrow 58 \quad-0.13531$

$53 \rightarrow 58 \quad 0.35444$

$54 \rightarrow 58 \quad-0.10275$

$56 \rightarrow 58 \quad 0.54232$

$54 \rightarrow 58 \quad 0.49279$

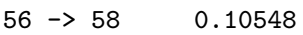

$57 \rightarrow 59 \quad-0.47052$

$57 \rightarrow 60 \quad 0.68746$

$57 \rightarrow 61 \quad 0.12760$

$54 \rightarrow 58 \quad 0.46373$

$57 \rightarrow 59 \quad 0.47150$

$57 \rightarrow 60 \quad-0.11225$

$57 \rightarrow 61 \quad 0.67966$

$57 \rightarrow 64 \quad-0.11149$ 


\section{$\mathrm{ORN}_{\mathrm{A}}$}

\begin{tabular}{|c|c|c|c|c|c|c|c|}
\hline \multicolumn{8}{|c|}{ B3LYP 6-31+G* } \\
\hline $1: 2.6480$ & $\mathrm{eV}$ & 468.22 & $\mathrm{~nm}$ & $f=0.8748$ & 71 & $->72$ & 0.56865 \\
\hline \multirow[t]{2}{*}{$2: 2.7853$} & $\mathrm{eV}$ & 445.14 & $\mathrm{~nm}$ & $f=0.0000$ & 70 & $->72$ & 0.67912 \\
\hline & & & & & 70 & $->73$ & -0.12395 \\
\hline \multirow[t]{2}{*}{$3: 3.6045$} & $\mathrm{eV}$ & 343.97 & $\mathrm{~nm}$ & $f=0.0313$ & 69 & $->72$ & 0.51596 \\
\hline & & & & & 71 & $->73$ & -0.44206 \\
\hline $4: 3.6236$ & $\mathrm{eV}$ & 342.16 & $\mathrm{~nm}$ & $f=0.0003$ & 68 & $->72$ & 0.69759 \\
\hline \multirow[t]{4}{*}{$5: 3.9919$} & $\mathrm{eV}$ & 310.59 & $\mathrm{~nm}$ & $f=0.1351$ & 66 & $->72$ & -0.16347 \\
\hline & & & & & 67 & $->72$ & 0.44564 \\
\hline & & & & & 69 & $->72$ & 0.28931 \\
\hline & & & & & 71 & $->73$ & 0.33209 \\
\hline \multirow[t]{4}{*}{$6: 4.0556$} & $\mathrm{eV}$ & 305.71 & $\mathrm{~nm}$ & $f=0.1361$ & 66 & $->72$ & 0.14619 \\
\hline & & & & & 67 & $->72$ & 0.52488 \\
\hline & & & & & 69 & $->72$ & -0.23054 \\
\hline & & & & & 71 & $->73$ & -0.28162 \\
\hline $7: 4.4650$ & $\mathrm{eV}$ & 277.68 & $\mathrm{~nm}$ & $f=0.0583$ & 71 & $->74$ & 0.67154 \\
\hline \multirow[t]{3}{*}{$8: 4.4881$} & $\mathrm{eV}$ & 276.25 & $\mathrm{~nm}$ & $f=0.0000$ & 70 & $->72$ & 0.14592 \\
\hline & & & & & 70 & $->73$ & 0.67513 \\
\hline & & & & & 70 & $->75$ & -0.11372 \\
\hline \multicolumn{8}{|c|}{ B3LYP $6-31+G *$} \\
\hline $1: 2.6480$ & $\mathrm{eV}$ & 468.22 & $\mathrm{~nm}$ & $f=0.8748$ & 71 & $->72$ & 0.56865 \\
\hline \multirow[t]{2}{*}{$2: 2.7853$} & $\mathrm{eV}$ & 445.14 & $\mathrm{~nm}$ & $f=0.0000$ & 70 & $->72$ & 0.67912 \\
\hline & & & & & 70 & -> 73 & -0.12395 \\
\hline \multirow[t]{2}{*}{$3: 3.6045$} & $\mathrm{eV}$ & 343.97 & $\mathrm{~nm}$ & $f=0.0313$ & 69 & $->72$ & 0.51596 \\
\hline & & & & & 71 & $->73$ & -0.44206 \\
\hline $4: 3.6236$ & $\mathrm{eV}$ & 342.16 & $\mathrm{~nm}$ & $f=0.0003$ & 68 & $->72$ & 0.69759 \\
\hline \multirow[t]{4}{*}{$5: 3.9919$} & $\mathrm{eV}$ & 310.59 & $\mathrm{~nm}$ & $f=0.1351$ & 66 & -> 72 & -0.16347 \\
\hline & & & & & 67 & $->72$ & 0.44564 \\
\hline & & & & & 69 & $->72$ & 0.28931 \\
\hline & & & & & 71 & $->73$ & 0.33209 \\
\hline \multirow[t]{4}{*}{$6: 4.0556$} & $\mathrm{eV}$ & 305.71 & $\mathrm{~nm}$ & $f=0.1361$ & 66 & $->72$ & 0.14619 \\
\hline & & & & & 67 & $->72$ & 0.52488 \\
\hline & & & & & 69 & $->72$ & -0.23054 \\
\hline & & & & & 71 & $->73$ & -0.28162 \\
\hline $7: 4.4650$ & $\mathrm{eV}$ & 277.68 & $\mathrm{~nm}$ & $f=0.0583$ & 71 & $->74$ & 0.67154 \\
\hline \multirow[t]{3}{*}{$8: 4.4881$} & $\mathrm{eV}$ & 276.25 & $\mathrm{~nm}$ & $f=0.0000$ & 70 & $->72$ & 0.14592 \\
\hline & & & & & 70 & $->73$ & 0.67513 \\
\hline & & & & & 70 & $->75$ & -0.11372 \\
\hline
\end{tabular}

B3LYP 6-31+G* PCM (methanol) [optimized with 6-31G* PCM (methanol)]

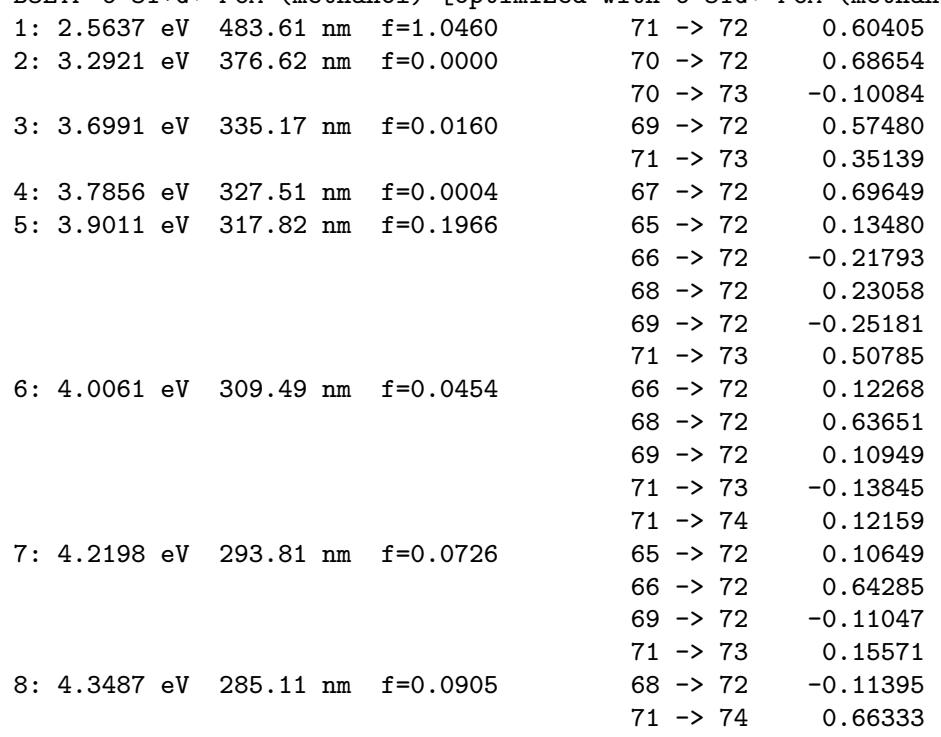


$\mathrm{ASF}_{\mathrm{A}}$

B3LYP $6-31+G *$

1: $2.5804 \mathrm{eV} \quad 480.49 \mathrm{~nm} \quad f=0.7969$

2: $2.8754 \mathrm{eV} \quad 431.19 \mathrm{~nm} \quad f=0.0000$

3: $3.4341 \mathrm{eV} \quad 361.04 \mathrm{~nm} \quad f=0.0001$

4: $3.5327 \mathrm{eV} \quad 350.97 \mathrm{~nm} \quad f=0.0135$

5: $3.7672 \mathrm{eV} \quad 329.11 \mathrm{~nm} \quad f=0.0003$

6: $3.8948 \mathrm{eV} \quad 318.33 \mathrm{~nm} \quad f=0.1480$

7: $3.9457 \mathrm{eV} \quad 314.22 \mathrm{~nm} \quad f=0.0000$

8: $3.9805 \mathrm{eV} \quad 311.48 \mathrm{~nm} \quad f=0.1488$

\begin{tabular}{|c|c|c|}
\hline 64 & $\rightarrow 6$ & 0.57170 \\
\hline 63 & $\rightarrow 6$ & 0.68131 \\
\hline 63 & $\rightarrow \epsilon$ & -0.11886 \\
\hline 59 & $\rightarrow \epsilon$ & -0.17434 \\
\hline 61 & $\rightarrow \epsilon$ & 0.66611 \\
\hline 62 & $\rightarrow \epsilon$ & 0.57014 \\
\hline 64 & $\rightarrow \epsilon$ & -0.36687 \\
\hline 59 & $\rightarrow \epsilon$ & 0.63435 \\
\hline 59 & $\rightarrow \epsilon$ & 0.16095 \\
\hline 61 & $\rightarrow \epsilon$ & 0.20097 \\
\hline 58 & $\rightarrow$ & 0.16919 \\
\hline 60 & $\rightarrow$ & 0.43762 \\
\hline 62 & $\rightarrow$ & 0.22458 \\
\hline 64 & $\rightarrow$ & 0.40300 \\
\hline 64 & $\rightarrow$ & 0.69209 \\
\hline 58 & $\rightarrow$ & -0.14525 \\
\hline 60 & $\rightarrow$ & 0.53132 \\
\hline & $\rightarrow$ & -0.18664 \\
\hline
\end{tabular}

$64 \rightarrow 66-0.31253$

$\begin{array}{llr}64 \rightarrow 66 & 0.59789 \\ 62 \rightarrow 65 & 0.68377 \\ 62 \rightarrow 66 & -0.10764 \\ 59 \rightarrow 65 & 0.19618 \\ 59 \rightarrow 66 & 0.11952 \\ 61 \rightarrow 65 & 0.64680 \\ 63 \rightarrow 65 & 0.60763 \\ 64 \rightarrow 66 & -0.28780 \\ 59 \rightarrow 65 & 0.63112 \\ 59 \rightarrow 66 & 0.11887 \\ 61 \rightarrow 65 & -0.23478 \\ 61 \rightarrow 66 & 0.10951 \\ 58 \rightarrow 65 & 0.15011 \\ 60 \rightarrow 65 & 0.40783 \\ 63 \rightarrow 65 & 0.16279 \\ 64 \rightarrow 66 & 0.47813 \\ 58 \rightarrow 65 & -0.12791 \\ 60 \rightarrow 65 & 0.55051 \\ 63 \rightarrow 65 & -0.15527 \\ 64 \rightarrow 66 & -0.31964 \\ 64 \rightarrow 67 & 0.67426\end{array}$




\section{$\mathrm{ZFP}_{\mathrm{A}}$}

B3LYP $631 \mathrm{G*}$

\begin{tabular}{|c|c|c|c|c|c|c|}
\hline $1: 2.6360$ & $\mathrm{eV}$ & $470.34 \mathrm{~nm}$ & $f=0.8595$ & 75 & $\rightarrow 76$ & 0.57358 \\
\hline & & & & 75 & -> 77 & 0.12154 \\
\hline $2: 2.8410$ & $\mathrm{eV}$ & $436.41 \mathrm{~nm}$ & $f=0.0000$ & 74 & $->76$ & 0.67894 \\
\hline & & & & 74 & -> 77 & -0.12713 \\
\hline $3: 3.6130$ & $\mathrm{eV}$ & $343.16 \mathrm{~nm}$ & $f=0.0684$ & 73 & $\rightarrow 76$ & -0.47956 \\
\hline & & & & 75 & -> 77 & 0.47570 \\
\hline $4: 3.6566$ & $\mathrm{eV}$ & $339.07 \mathrm{~nm}$ & $f=0.0005$ & 72 & $\rightarrow 76$ & 0.69524 \\
\hline $5: 3.9687$ & $\mathrm{eV}$ & $312.40 \mathrm{~nm}$ & $f=0.2256$ & 69 & $\rightarrow 76$ & -0.20514 \\
\hline & & & & 71 & $->76$ & -0.17874 \\
\hline & & & & 73 & $\rightarrow 76$ & 0.41069 \\
\hline & & & & 75 & $->77$ & 0.39092 \\
\hline $6: 4.1003$ & $\mathrm{eV}$ & $300.38 \mathrm{~nm}$ & $f=0.0311$ & 71 & $\Rightarrow 76$ & 0.66487 \\
\hline & & & & 75 & $\rightarrow 77$ & 0.10594 \\
\hline $7: 4.2150$ & $\mathrm{eV}$ & $294.15 \mathrm{~nm}$ & $f=0.0009$ & 70 & $\rightarrow 76$ & 0.66317 \\
\hline & & & & 70 & -> 77 & 0.17338 \\
\hline $8: 4.4287$ & $\mathrm{eV}$ & $279.95 \mathrm{~nm}$ & $f=0.0615$ & 75 & $\rightarrow 78$ & 0.66963 \\
\hline B3LYP 631 & $+\mathrm{G} *$ & & & & & \\
\hline 1: 2.5672 & $\mathrm{eV}$ & $482.95 \mathrm{~nm}$ & $f=0.8587$ & 75 & $\rightarrow 76$ & 0.58139 \\
\hline $2: 2.9731$ & $\mathrm{eV}$ & $417.01 \mathrm{~nm}$ & $f=0.0000$ & 74 & $\rightarrow 76$ & 0.67990 \\
\hline & & & & 74 & $\rightarrow 78$ & -0.11038 \\
\hline $3: 3.0959$ & $\mathrm{eV}$ & $400.47 \mathrm{~nm}$ & $f=0.0386$ & 75 & -> 77 & 0.69091 \\
\hline $4: 3.5673$ & $\mathrm{eV}$ & $347.56 \mathrm{~nm}$ & $f=0.0695$ & 73 & $\rightarrow 76$ & -0.39095 \\
\hline & & & & 75 & $\rightarrow 78$ & 0.54429 \\
\hline & & & & 75 & $\rightarrow 79$ & -0.13130 \\
\hline $5: 3.6674$ & $\mathrm{eV}$ & $338.07 \mathrm{~nm}$ & $f=0.0017$ & 73 & $\rightarrow 76$ & -0.26781 \\
\hline & & & & 75 & $\rightarrow 79$ & 0.63916 \\
\hline $6: 3.6870$ & $\mathrm{eV}$ & $336.27 \mathrm{~nm}$ & $f=0.0006$ & 72 & $\rightarrow 76$ & 0.69463 \\
\hline $7: 3.7829$ & $\mathrm{eV}$ & $327.75 \mathrm{~nm}$ & $f=0.0014$ & 75 & $\rightarrow 80$ & -0.35516 \\
\hline & & & & 75 & $\rightarrow>81$ & 0.57628 \\
\hline $8: 3.8133$ & $\mathrm{eV}$ & $325.14 \mathrm{~nm}$ & $f=0.0041$ & 75 & $\rightarrow 80$ & 0.58586 \\
\hline & & & & 75 & $->81$ & 0.37368 \\
\hline 3LYP 631 & $+\mathrm{G} *$ & PCM (methar & & & & \\
\hline $1: 2.6223$ & $\mathrm{eV}$ & $472.81 \mathrm{~nm}$ & $f=1.0830$ & 75 & $\rightarrow 76$ & 0.60548 \\
\hline $2: 3.4384$ & $\mathrm{eV}$ & $360.59 \mathrm{~nm}$ & $f=0.0000$ & 73 & $\rightarrow 76$ & 0.68682 \\
\hline $3: 3.6365$ & $\mathrm{eV}$ & $340.94 \mathrm{~nm}$ & $f=0.0008$ & 72 & $\rightarrow 76$ & 0.66926 \\
\hline & & & & 72 & -> 77 & 0.14326 \\
\hline $4: 3.7532$ & $\mathrm{eV}$ & $330.34 \mathrm{~nm}$ & $f=0.0152$ & 74 & $\rightarrow 76$ & 0.57502 \\
\hline & & & & 75 & -> 77 & 0.34773 \\
\hline $5: 3.8883$ & $\mathrm{eV}$ & $318.86 \mathrm{~nm}$ & $f=0.0026$ & 71 & $\rightarrow 76$ & 0.68200 \\
\hline $6: 3.9399$ & $\mathrm{eV}$ & $314.69 \mathrm{~nm}$ & $f=0.2041$ & 69 & $\rightarrow 76$ & 0.20515 \\
\hline & & & & 70 & $\rightarrow 76$ & 0.11222 \\
\hline & & & & 74 & $\rightarrow 76$ & -0.27900 \\
\hline & & & & 75 & $\rightarrow 77$ & 0.54826 \\
\hline $7: 4.1378$ & $\mathrm{eV}$ & $299.64 \mathrm{~nm}$ & $f=0.0095$ & 70 & $\Rightarrow 76$ & 0.59724 \\
\hline & & & & 75 & $->78$ & 0.32597 \\
\hline 8: 4.3104 & $\mathrm{eV}$ & $287.64 \mathrm{~nm}$ & $f=0.1014$ & 70 & $\rightarrow 76$ & -0.30680 \\
\hline & & & & 75 & $->78$ & 0.58562 \\
\hline & & & & 75 & $\rightarrow 79$ & 0.11135 \\
\hline
\end{tabular}




\section{$\operatorname{DSR}_{\mathrm{A}}$}

B3LYP $631 \mathrm{G*}$

1: $2.4252 \mathrm{eV} \quad 511.24 \mathrm{~nm} \quad f=0.9336$

$\begin{array}{rr}75 \rightarrow 76 & 0.55989 \\ 74 \rightarrow 76 & 0.67570 \\ 74 \rightarrow 77 & 0.14280 \\ 71 \rightarrow 76 & -0.45346 \\ 71 \rightarrow 77 & 0.11700 \\ 72 \rightarrow 76 & 0.50984 \\ 73 \rightarrow 76 & 0.54397 \\ 75 \rightarrow 77 & 0.41613 \\ 71 \rightarrow 76 & 0.49445 \\ 71 \rightarrow 77 & -0.10444 \\ 72 \rightarrow 76 & 0.47448 \\ 69 \rightarrow 76 & -0.22281 \\ 70 \rightarrow 76 & -0.35476 \\ 73 \rightarrow 76 & -0.28597 \\ 75 \rightarrow 77 & 0.39091 \\ 69 \rightarrow 76 & -0.14036 \\ 70 \rightarrow 76 & 0.59028 \\ 73 \rightarrow 76 & -0.16007 \\ 75 \rightarrow 77 & 0.23345 \\ 74 \rightarrow 76 & -0.16391 \\ 74 \rightarrow 77 & 0.66861\end{array}$

B3LYP $631+G *$

1: $2.3639 \mathrm{eV} \quad 524.48 \mathrm{~nm} \quad f=0.9581$

$75->76$

0.56817

2: $2.7435 \mathrm{eV} \quad 451.92 \mathrm{~nm} \quad f=0.0000$

$74 \rightarrow 76$

$74 \rightarrow 77$

3: $3.2446 \mathrm{eV} \quad 382.13 \mathrm{~nm} \quad \mathrm{f}=0.0192$

$73 \rightarrow 76$

0.67719

$75 \rightarrow 77$

$-0.14220$

4: $3.2887 \mathrm{eV} \quad 377.01 \mathrm{~nm} \quad \mathrm{f}=0.0008$

$71 \rightarrow 76$

0.53778

$\begin{array}{lll}71-77 & 0.11489\end{array}$

$72 \rightarrow 76 \quad 0.53584$

5: $3.3542 \mathrm{eV} \quad 369.64 \mathrm{~nm} \quad f=0.0041$

0.52055

$\begin{array}{lll}71 \rightarrow 77 & 0.10506\end{array}$

6: $3.6906 \mathrm{eV} \quad 335.94 \mathrm{~nm} \quad f=0.2326$

$72 \rightarrow 76$

$-0.44403$

$69 \rightarrow 76 \quad 0.21221$

$\begin{array}{lll}70 \rightarrow 76 & 0.26424\end{array}$

$73 \rightarrow 76 \quad 0.32036$

$\begin{array}{lll}75 & \rightarrow 77 & 0.42120\end{array}$

7: $3.8017 \mathrm{eV} \quad 326.13 \mathrm{~nm} \quad \mathrm{f}=0.0600$

$70 \rightarrow 76 \quad 0.63395$

$73 \rightarrow 76 \quad-0.11944$

$75 \rightarrow 77 \quad-0.16525$

8: $3.8849 \mathrm{eV} \quad 319.15 \mathrm{~nm} \quad f=0.0143$

$75->78$

0.67910

B3LYP 631+G* PCM (methanol)

1: $2.2948 \mathrm{eV} \quad 540.29 \mathrm{~nm} \quad f=1.0857$

2: $3.0984 \mathrm{eV} \quad 400.15 \mathrm{~nm} \quad f=0.0000$

$75 \rightarrow 76$

0.59737

$73 \rightarrow 76 \quad 0.67991$

$73 \rightarrow 77 \quad-0.13467$

3: $3.2311 \mathrm{eV} \quad 383.72 \mathrm{~nm} \quad f=0.0020$

$72 \rightarrow 76$

0.66380

$72 \rightarrow 77 \quad 0.15594$

4: $3.3324 \mathrm{eV} \quad 372.06 \mathrm{~nm} \quad f=0.0307$

$74 \rightarrow 76$

0.53454

$75->77 \quad-0.42692$

5: $3.4831 \mathrm{eV} \quad 355.96 \mathrm{~nm} \quad f=0.0037$

6: $3.6460 \mathrm{eV} \quad 340.06 \mathrm{~nm} \quad f=0.2786$

7: $3.7963 \mathrm{eV} \quad 326.59 \mathrm{~nm} \quad f=0.0485$

$71 \rightarrow 76$

0.68436

$69 \rightarrow 76 \quad 0.20202$

$70 \rightarrow 76 \quad 0.19352$

$74 \rightarrow 76 \quad 0.34712$

$75 \rightarrow 77 \quad 0.45906$

$70 \rightarrow 76 \quad 0.66048$

$75 \rightarrow 77 \quad-0.12097$

$68 \rightarrow 76 \quad 0.62482$

$68 \rightarrow 77 \quad 0.13676$

$69 \rightarrow 76 \quad-0.21082$ 


\section{$\mathrm{KAE}_{\mathrm{A}}$}

\begin{tabular}{|c|c|c|c|c|c|c|c|}
\hline \multicolumn{8}{|c|}{ B3LYP $6-31 \mathrm{G} *$} \\
\hline \multirow[t]{2}{*}{$1: 2.2459$} & $\mathrm{eV}$ & 552.05 & $\mathrm{~nm}$ & $f=0.8227$ & 77 & $->78$ & 0.57171 \\
\hline & & & & & 77 & $->79$ & 0.10858 \\
\hline \multirow[t]{2}{*}{$2: 2.6585$} & $\mathrm{eV}$ & 466.36 & $\mathrm{~nm}$ & $f=0.0000$ & 76 & $->78$ & 0.67384 \\
\hline & & & & & 76 & $->79$ & -0.16055 \\
\hline \multirow[t]{2}{*}{$3: 3.1782$} & $\mathrm{eV}$ & 390.11 & $\mathrm{~nm}$ & $f=0.1435$ & 75 & $->78$ & 0.45790 \\
\hline & & & & & 77 & $->79$ & 0.50167 \\
\hline $4: 3.5658$ & $\mathrm{eV}$ & 347.70 & $\mathrm{~nm}$ & $f=0.0004$ & 74 & $->78$ & 0.69510 \\
\hline \multirow[t]{4}{*}{$5: 3.7379$} & $\mathrm{eV}$ & 331.70 & $\mathrm{~nm}$ & $f=0.2563$ & 72 & $->78$ & -0.31275 \\
\hline & & & & & 75 & $->78$ & 0.41754 \\
\hline & & & & & 77 & -> 79 & -0.32023 \\
\hline & & & & & 77 & $->80$ & -0.11223 \\
\hline $6: 3.8490$ & $\mathrm{eV}$ & 322.12 & $\mathrm{~nm}$ & $f=0.0104$ & 73 & $->78$ & 0.68521 \\
\hline \multirow[t]{2}{*}{$7: 3.9923$} & $\mathrm{eV}$ & 310.56 & $\mathrm{~nm}$ & $f=0.0000$ & 76 & $->78$ & 0.18237 \\
\hline & & & & & 76 & $->79$ & 0.66893 \\
\hline \multirow[t]{2}{*}{$8: 4.2169$} & $\mathrm{eV}$ & 294.02 & $\mathrm{~nm}$ & $f=0.0399$ & 72 & $->78$ & -0.18330 \\
\hline & & & & & 77 & $\rightarrow>80$ & 0.65847 \\
\hline \multicolumn{8}{|c|}{ B3LYP $6-31+G *$} \\
\hline \multirow[t]{2}{*}{$1: 2.1471$} & $\mathrm{eV}$ & 577.44 & $\mathrm{~nm}$ & $f=0.7939$ & 77 & $->78$ & 0.58075 \\
\hline & & & & & 77 & $\rightarrow>80$ & 0.10328 \\
\hline $2: 2.6359$ & $\mathrm{eV}$ & 470.36 & $\mathrm{~nm}$ & $f=0.0001$ & 77 & $->79$ & 0.70324 \\
\hline \multirow[t]{2}{*}{$3: 2.7686$} & $\mathrm{eV}$ & 447.81 & $\mathrm{~nm}$ & $f=0.0000$ & 76 & $->78$ & 0.67465 \\
\hline & & & & & 76 & $\rightarrow>80$ & -0.16355 \\
\hline \multirow{2}{*}{$4: 3.0996$} & $\mathrm{eV}$ & 400.00 & $\mathrm{~nm}$ & $f=0.1706$ & 75 & $->78$ & 0.42929 \\
\hline & & & & & 77 & $\rightarrow>80$ & 0.52456 \\
\hline $5: 3.3976$ & $\mathrm{eV}$ & 364.91 & $\mathrm{~nm}$ & $f=0.0011$ & 77 & $->81$ & 0.69959 \\
\hline $6: 3.5781$ & $\mathrm{eV}$ & 346.50 & $\mathrm{~nm}$ & $f=0.0005$ & 74 & $->78$ & 0.69464 \\
\hline \multirow[t]{4}{*}{$7: 3.6040$} & $\mathrm{eV}$ & 344.02 & $\mathrm{~nm}$ & $f=0.2555$ & 72 & $->78$ & -0.24890 \\
\hline & & & & & 75 & $->78$ & 0.45616 \\
\hline & & & & & 77 & $->80$ & -0.30580 \\
\hline & & & & & 77 & $->82$ & 0.15439 \\
\hline \multirow[t]{3}{*}{$8: 3.7861$} & $\mathrm{eV}$ & 327.47 & $\mathrm{~nm}$ & $f=0.0002$ & 77 & $->83$ & 0.61267 \\
\hline & & & & & 77 & $->84$ & -0.30141 \\
\hline & & & & & 77 & $->85$ & 0.15226 \\
\hline \multicolumn{2}{|c|}{ B3LYP $6-31+G *$} & \multicolumn{6}{|c|}{ PCM (methanol) } \\
\hline $1: 2.2777$ & $\mathrm{eV}$ & 544.33 & $\mathrm{~nm}$ & $f=0.9791$ & 77 & $->78$ & 0.61100 \\
\hline \multirow[t]{2}{*}{$2: 3.2310$} & $\mathrm{eV}$ & 383.73 & $\mathrm{~nm}$ & $f=0.1164$ & 76 & $->78$ & 0.40097 \\
\hline & & & & & 77 & $->79$ & 0.56052 \\
\hline \multirow[t]{2}{*}{$3: 3.3854$} & $\mathrm{eV}$ & 366.23 & $\mathrm{~nm}$ & $f=0.0000$ & 75 & $->78$ & 0.68049 \\
\hline & & & & & 75 & -> 79 & -0.13981 \\
\hline \multirow[t]{3}{*}{$4: 3.7155$} & $\mathrm{eV}$ & 333.69 & $\mathrm{~nm}$ & $f=0.4097$ & 74 & $->78$ & -0.28943 \\
\hline & & & & & 76 & $->78$ & 0.47307 \\
\hline & & & & & 77 & $->79$ & -0.28242 \\
\hline \multirow[t]{2}{*}{$5: 3.8239$} & $\mathrm{eV}$ & 324.23 & $\mathrm{~nm}$ & $f=0.0006$ & 72 & $->78$ & 0.43122 \\
\hline & & & & & 73 & $->78$ & 0.54251 \\
\hline $6: 3.8902$ & $\mathrm{eV}$ & 318.71 & $\mathrm{~nm}$ & $f=0.4891$ & 74 & $->78$ & 0.60613 \\
\hline & & & & & 76 & $->78$ & 0.20234 \\
\hline & & & & & 77 & -> 79 & -0.16352 \\
\hline $7: 4.0462$ & $\mathrm{eV}$ & 306.42 & $\mathrm{~nm}$ & $f=0.0126$ & 72 & $->78$ & 0.45080 \\
\hline & & & & & 73 & $->78$ & -0.35835 \\
\hline & & & & & 77 & $->80$ & 0.37365 \\
\hline $8: 4.1497$ & $\mathrm{eV}$ & 298.78 & $\mathrm{~nm}$ & $f=0.0008$ & 77 & $->81$ & 0.67505 \\
\hline & & & & & 77 & $->82$ & -0.18689 \\
\hline
\end{tabular}

B3LYP 6-31+G* PCM (methanol) [optimized with 6-31G* PCM (methanol)]

1: $2.3076 \mathrm{eV} 537.29 \mathrm{~nm} f=0.9429$

2: $3.2553 \mathrm{eV} \quad 380.87 \mathrm{~nm} \quad f=0.1224 \quad 76 \rightarrow 78 \quad-0.42718$

$\begin{array}{lll}77 \rightarrow 79 & 0.54289\end{array}$

3: $3.3595 \mathrm{eV} \quad 369.06 \mathrm{~nm} \quad \mathrm{f}=0.0000 \quad 75 \rightarrow 78 \quad 0.68232$

$4: 3.6972 \mathrm{eV} \quad 335.35 \mathrm{~nm} f=0.4418 \quad 0.26191$ 
5: $3.8170 \mathrm{eV} \quad 324.82 \mathrm{~nm} \quad \mathrm{f}=0.0006$

$\begin{array}{lr}76 \rightarrow>78 & 0.46277 \\ 77 \rightarrow 79 & 0.32137 \\ 72 \rightarrow 78 & 0.69228 \\ 74 \rightarrow 78 & 0.61679 \\ 76 \rightarrow 78 & 0.17021 \\ 77 \rightarrow 79 & 0.16153 \\ 73 \rightarrow 78 & 0.63000 \\ 77 \rightarrow 80 & -0.27537 \\ 73 \rightarrow 78 & 0.27353 \\ 77 \rightarrow 80 & 0.61507\end{array}$

Permanent and transition dipole moments for CFP (gas phase, 6-31G*)

\begin{tabular}{|l|r|r|r|r|}
\hline & $\mu_{x}$ & $\mu_{y}$ & $\mu_{z}$ & $|\mu|$ \\
\hline $\bar{\mu}=\langle 0|\mu| 0\rangle$ & -3.6952 & -0.1602 & -2.7317 & 4.5981 \\
$\langle 1|\mu| 1\rangle-\bar{\mu}$ & -1.7894 & -0.0984 & 1.7383 & 2.4966 \\
$\langle 2|\mu| 2\rangle-\bar{\mu}$ & 1.4173 & 0.0597 & -3.8054 & 4.0613 \\
$\langle 3|\mu| 3\rangle-\bar{\mu}$ & -3.4892 & -0.1552 & 3.6603 & 5.0592 \\
$\langle 4|\mu| 4\rangle-\bar{\mu}$ & -3.0044 & -0.1364 & 2.6167 & 3.9865 \\
$\langle 5|\mu| 5\rangle-\bar{\mu}$ & 5.0631 & 0.2185 & -4.3567 & 6.6830 \\
\hline$\langle 1|\mu| 2\rangle$ & 0.0295 & -0.0354 & 0.0605 & 0.0761 \\
$\langle 1|\mu| 3\rangle$ & 1.8217 & 0.0774 & -1.9602 & $\mathbf{2 . 6 7 7 2}$ \\
$\langle 2|\mu| 3\rangle$ & -0.0356 & -0.0533 & 0.0492 & 0.0808 \\
$\langle 1|\mu| 4\rangle$ & -2.6909 & -0.1220 & 2.4560 & $\mathbf{3 . 6 4 5 3}$ \\
$\langle 2|\mu| 4\rangle$ & 0.0899 & 0.0735 & -0.1034 & 0.1555 \\
$\langle 3|\mu| 4\rangle$ & -3.3651 & -0.1506 & 3.6776 & $\mathbf{4 . 9 8 7 1}$ \\
$\langle 1|\mu| 5\rangle$ & 0.1829 & 0.0100 & -0.2274 & 0.2920 \\
$\langle 2|\mu| 5\rangle$ & -0.1371 & 0.0057 & 0.14732 & 0.2013 \\
$\langle 3|\mu| 5\rangle$ & 3.7596 & 0.1706 & -3.8291 & $\mathbf{5 . 3 6 9 0}$ \\
$\langle 4|\mu| 5\rangle$ & 0.6781 & 0.0226 & -0.5157 & 0.8522 \\
\hline
\end{tabular}

